\title{
Multiple Parameters and Target Optimization of Splitter Blades for Blood Pumps Using CFD and Neural Networks
}

\section{Zheqin Yu ( $\nabla$ yzq01113@163.com )}

Central south university

Jianping Tan

central south univercity

\section{Shuai Wang}

central south univercity

\section{Research}

Keywords: Blood pump, Impeller, Splitter blades, Multiple parameters and objectives optimization, CFD, Neural Networks, PIV

Posted Date: February 25th, 2020

DOI: https://doi.org/10.21203/rs.2.24520/v1

License: (c) (i) This work is licensed under a Creative Commons Attribution 4.0 International License. Read Full License 


\section{Abstract}

Background: The splitter blade is an improved structure effective in traditional pumps. However, applying splitter blades to blood pumps is a complex optimization problem with multiple parameters and objectives, because structural parameters, blood circulation dynamics, and blood damage need to be considered simultaneously. This study aims to obtain the performance impact of the splitter blade and make it well used in blood pumps through CFD and neural networks.

Results: This study combines CFD and neural networks. And hydraulic experiments and PIV technology were used. In the optimization study, the number of blades, axial length, and circumferential offset are optimization parameters, and hydraulic performance and hemolytic prediction index are optimization targets. The study analyzes the influence of each parameter on performance and completes the optimization of the parameters. In the results, the optimal parameters of the number of blades, axial length ratio, and circumferential offset are $2,6^{\circ}$, and 0.41 , respectively. Under optimized parameters, hydraulic performance can be significantly improved. And the results of hemolysis prediction and micro PIV experiments reflect that there is no increase in the risk of hemolytic damage.

Conclusion: This study provides a method and ideas for improving the structure of the blood pump. The established optimization method can be effectively applied to the design and research of blood pumps with complex, high precision, and multiple parameters and targets.

\section{Background}

Heart failure and lesions are diseases that seriously endanger the human body, and these diseases are often irreversible. At present, heart transplantation is a feasible method, but there is a shortage of heart donors[1]. Therefore, implanting VAD has gradually become a popular method of treatment. The blood pump is the core component responsible for generating blood circulation energy in VAD, and its design directly affects the health of patients[2,3].

The working mechanism of the blood pump is similar to that of industrial water pumps, and the circulating energy of the fluid is generated by the high-speed rotation of the impeller. However, the working medium of the blood pump is blood, so the design must ensure that it does not cause excessive damage to the blood. The blood damage caused by the blood pump is thought to be mainly caused by the shear stress in the flow field[2,4,5]. Because the impeller is the core structure that generates fluid energy and affects the flow field, the structural design of the impeller is directly related to the performance of the blood pump.

The structural design of the blood pump has strict requirements, and each structural part needs to be carefully designed, and analyzed, Even the improvement of small structures can often improve the performance of blood pumps[3]. Splitter blades are an improved structure commonly used in industrial water pumps. These blades are arranged between every two main blades. Their primary function is to improve the work performance of the impeller and stabilize the flow in the flow field[6,7]. However, the 
application of splitter blades in blood pumps is still incomplete. In the design and application, it is necessary to ensure that the splitter blades will not cause additional blood damage, and then consider its effect on improving circulation performance.

The design of a blood pump requires consideration of both circulatory and hemolytic properties. In the research and design phase, CFD simulation is the most effective and versatile method. In the CFD simulation, numerical calculations are performed on the blood pump model, and the calculated results are used to estimate the circulation performance and blood damage[8-10]. However, there are many structural parameters of blood pumps, and the influencing characteristics of each parameter are often non-linear, and the parameters may also interact with each other[11,12]. Using only CFD simulation to determine the optimal parameters of the structure will require a lot of calculations, which will lead to a significant increase in time cost. And it is difficult for such a method to obtain sufficiently accurate structural parameters.

The neural network method for performance estimation is an effective aided design method, which has been well implemented in many fields [13-15]. The neural network is a machine learning method that simulates biological nerves. It can form an accurate prediction model after training on a certain number of samples[16,17]. This method can significantly reduce the time requirements for simulation and experiment. This method is especially suitable for multi-parameter and multi-objective optimization research, which is consistent with the design requirements of blood pumps.

In this study, the research purpose is to optimize and apply the splitter blade in the blood pump. Aiming at the design requirements of multiple parameters and objectives, this study established an optimization method combining CFD and neural networks. The experiments in the research include hydraulic experiments and micro PIV experiments. The performance impact of the splitter blades parameters was obtained in the study, and the optimization of the parameters was performed to improve hydraulic performance without affecting blood damage.

\section{Results}

\section{Parameter characteristic analysis and establishment of the neural network prediction model}

To achieve the precise optimization of the splitter blades parameters, a BP neural network is used to establish the mapping relationship between parameters and performance. At the design stage, performance prediction under different parameters is performed by neural networks. There are three input parameters of the neural network, which are the number of blades, the axial length ratio, and the circumferential offset. The analysis range of the input parameters is determined through previous theoretical analysis and simulation estimation. The splitter blades are arranged between every two main blades. The input analysis variables for the number of blades are 0,2 , and 4 . The input range of the circumferential length is 0.3 to 0.7 , and the input range of the circumferential offset is $-15^{\circ}$ to $30^{\circ}$. 
The performance prediction calculation of each parameter was performed by CFD simulation, and the influence characteristics of each parameter on the performance of the blood pump were analyzed. Figure 1 shows the average performance results of the hydraulic head and hemolysis index under each parameter value, which reflects the influence of each parameter on performance. To compare the optimization effects of the splitter blades, the calculation of the splitter blades-free model was performed under the same conditions, the hydraulic head and hemolysis index of the model were $12.71 \mathrm{kPa}$ and $6.49 \times 10^{-3}$, respectively.

In the parameter analysis results, the number of blades has the most significant effect on performance. The average hydraulic heads of the 2 and 4 splitter blades models were improved by $0.44 \mathrm{kPa}$ and 0.98 $\mathrm{kPa}$ respectively compared to the structure without the splitter blades. However, the increase in the number of blades also increased the hemolysis index, especially the average hemolysis index of the four splitter blades model increased by $25 \%$. This makes no matter how the other two parameters are changed. The hemolysis index will be significantly improved under the 4 splitter blades. In addition, the hydraulic head curve under the axial length ratio and the circumferential offset degree both rises first and then decrease, and there are parameters that can make the hydraulic head optimal. For the hemolytic index, the axial length ratio will continue to increase with the increase of the ratio, and the hemolytic index will not be affected with a smaller axial length ratio. The hemolytic index of the circumferential offset degree decreases first and then increases. In general, when the axial length ratio is 0.3 to 0.4 , and the circumferential offset is $-15^{\circ}$ to $15^{\circ}$, the hemolysis index is not affected. The parameter performance impact analysis shows the possibility of the optimal performance of the splitter blade. Besides, there is a clear rule for the influence of each parameter on performance, which is conducive to achieving highprecision neural network training.

Use the data from the above simulation results to train the BP neural network. To avoid the impact of different data magnitudes on the accuracy, the sample data is normalized. In the established neural network, there are three input neurons and two output neurons. The number of hidden neurons in the middle is set to 12 , and the S-tangent function tansig is used as the excitation function of the hidden layer.

\section{Multi-objective optimization and results}

There are two main performance indicators of blood pumps, which are hydraulic performance and hemolytic performance. The purpose of parameter optimization is to improve hydraulic performance without improving hemolysis. Splitter blades are affected by multiple parameters, and the interaction between the parameters is not clear. Therefore, parameter optimization of blood pump splitter blades is a typical multi-objective optimization problem.

In the optimization, the performance of the blood pump is predicted by the trained BP neural network. For optimization purposes, the expectation of hydraulic performance is that the pressure head is as high as possible. The requirement of the hemolysis index is not higher than the model without splitter blades, and 
it is set to not higher than $6.49 \times 10^{-3}$ in the optimization model. The established multi-objective optimization model is:

$$
\left\{\begin{array}{c}
f_{1}\left(x_{1}, x_{2}, x_{3}\right) \Rightarrow \max \\
f_{2}\left(x_{1}, x_{2}, x_{3}\right) \leq 6.49 \times 10^{-3}
\end{array}\right.
$$

Figure 2 is the parameter optimization process of the splitter blades. In the optimization results, the number of splitter blades, axial length ratio, and circumferential offset is $2,0.41$, and $6^{\circ}$, respectively. Under this parameter, the hydraulic head predicted by the neural network is $13.13 \mathrm{kPa}$, and the hemolysis index is $6.49 \times 10^{-3}$. Its hydraulic head is higher than that without the splitter blades model, and the hemolysis index is equal to that without the splitter blades model, which meets the requirements of the optimization model. The performance results of the simulation under the optimized parameters are 13.14 $\mathrm{kPa}$ and $6.49 \times 10^{-3}$, which are consistent with the predicted values of the neural network model, which also reflects the accuracy of the neural network prediction.

\section{Experimental analysis}

To demonstrate the reliability of research and optimization, hydraulic experiments and PIV microflow experiments were performed. Hydraulic experiments are used to determine the effect of optimization improvements and to verify the accuracy of the simulation. The PIV microscopic experiment mainly measures and analyzes the flow inside the pump, and uses the results to analyze and evaluate whether there is a bad flow or hemolysis risk.

In hydraulic experiments, the pressure head and flow rate of the model under different impeller rotation speeds were measured. The experimental rotation speed range was from $2000 \mathrm{rpm}$ to $10000 \mathrm{rpm}$, and a set of measurements was performed every $1000 \mathrm{rpm}$. The flow measurement is the position behind the pump outlet, and the pressure head is the pump outlet pressure minus the inlet pressure. There are two models used in hydraulic experiments, one is model-a without splitter blades, and the other is model-b with optimized splitter blades. Except for the splitter blades structure, the other parameters of the two models remain the same.

Figure 3 is the performance curve of the pump measured by hydraulic experiments. In the results, graphs (a) and (b) are the experimental results of model-a and model-b, respectively. Similar to the simulation results, model-b with splitter blades can achieve higher pressure heads at the same flow rate. Model-b has pressure heads of $13.16 \mathrm{kPa}$ and $14.67 \mathrm{kPa}$ at $8000 \mathrm{rpm}$ and $10000 \mathrm{rpm}$, respectively. The head is 0.41 $\mathrm{kPa}$ and $0.89 \mathrm{kPa}$ higher than model-a. Besides, model-b has a better performance increase trend than model-a at 8000-10000rpm. The above results all show the effectiveness of splitter blades on performance improvement. Comparing the simulation and experimental results under design conditions, the flow difference is less than $5.0 \%$, and the head difference is less than $1.0 \%$, which also proves the reliability of the simulation results. 
In PIV microscopic experiments, the internal flow of the optimized blood pump prototype was measured. The measured positions include the inflow region of the front diffuser, the outflow region of the back diffuser, and the impeller region of two typical rotating areas, which correspond to the results of Figure 4, 5 , and 6 , respectively. In the velocity range of the flow field, the flow velocity in the inflow region and the outflow region is significantly lower than the impeller region. Their maximum velocity is about 1 to 1.5 $\mathrm{m} / \mathrm{s}$, while the maximum velocity in the impeller region is higher than $3 \mathrm{~m} / \mathrm{s}$.

The PIV results clearly reflect the differences in flow conditions between regions. The flow in the inflow area is stable and uniform. There is only a slight decrease in flow velocity near the wall and the front diffuser, but both are in the flow velocity range of 0.6 to $0.9 \mathrm{~m} / \mathrm{s}$. The flow in the outflow area has an apparent high-velocity area with a maximum velocity close to $1.5 \mathrm{~m} / \mathrm{s}$, which is caused by the fluid entering the outflow area after being accelerated by the impeller.

The impeller generates energy for fluid flow through high-speed rotation, so the flow in this area is also the most complicated. In Figure 6, (a) and (b) are the PIV results for two typical rotational positions. The velocity range of the impeller region is extensive, and the velocity ranges from 0 to $3 \mathrm{~m} / \mathrm{s}$. On the inflow side, the fluid enters the blade area near the pump casing wall with high velocity and reaches the highest flow velocity in the middle of the impeller. The flow gradually decreases and stabilizes in the rear region of the blade. In general, the flow in the impeller area is ideal, there is no apparent high-velocity area, but there is a low-velocity flow in the front area of each blade tip.

\section{Discussions}

The improvement of the blood pump structure needs to consider both the hydraulic performance and hemolytic damage, which is an intricate multi-parameter and multi-target design work. In this regard, CFD simulation and neural networks are combined. The prediction model of parameters and performance was established by the neural networks, and the optimal design of the structure parameters of splitter blades was carried out. Research is supplemented by hydraulic experiments and micro PIV experiments. Through the above work, the application of the splitter blades structure to the blood pump was studied.

According to the structural characteristics of splitter blades, there are three input parameters for this optimization, which are the number of split blades, axial length, and circumferential offset. There are two output indicators of pump performance, which are pressure head and hemolysis index. A performance prediction model was established by BP neural network, which has three input neurons, 12 hidden neurons, and two output neurons. Among them, the neural network uses the S-tangent function tansig as the excitation function of the hidden layer.

The CFD simulation calculation and analysis are performed on the models with different splitter blades parameters, and the simulation results are used as training samples for the neural network. In the simulation, the number of blade parameters is 0,2 , and 4 , the analysis range of the circumferential length is 0.3 to 0.7 , and the analysis range of the circumferential offset is $-15^{\circ}$ to $30^{\circ}$. 
The influence characteristics of the parameters are analyzed by simulation results. The results in Figure 1 show the effects of various parameters on performance. In the results, the number of blades has a stronger impact than other parameters, and increasing the number of blades can significantly improve hydraulic performance. This is because the splitter blades can exert additional force on the fluid. However, when the splitter blades were increased from 2 to 4 , the hemolytic performance deteriorated severely, which may be caused by excessive flow velocity changes after dividing too many channels. Besides, both blade length and offset can improve hydraulic performance without affecting hemolysis. The analysis results of parameter characteristics show the feasibility of splitter blades to enhance the performance of blood pumps.

The neural network is trained to establish the relationship between the splitter blades parameters and the blood pump performance, which is used to predict performance in optimization. In the optimization, there are two performance indicators, which are the pressure head and the hemolysis index. Among them, the expectation of the pressure head is as high as possible. Because the blood circulation requirement can be adjusted by reducing the impeller speed, it is also beneficial to reduce the risk of hemolysis. The requirement of the hemolysis index is not to increase, and the hemolysis index of the optimized structure cannot be higher than the original model without splitter blades. In the optimization results, the number of splitter blades is two, the axial length ratio is 0.41 , and the circumferential offset is $6^{\circ}$. Compared with the original model, the hydraulic head has increased by $0.43 \mathrm{kPa}$, and the hemolysis index has not increased, and the set optimization goal has been achieved.

In the study, the internal flow of the blood pump was measured and analyzed by PIV technology. In general, the flow state is stable, and there is no apparent hazardous flow state, but there are also several points worth noting. First, there is an area with a high velocity near the back diffuser. The reason is that the fluid has a large flow velocity after it is accelerated by the impeller, and then this state occurs after the fluid enters the back diffuser area. But because the flow velocity in this area is low, the risk of blood damage will not be excessively increased due to the injury mechanism[4,5]. Secondly, there will be a lowvelocity area in front of the blade tip. Because this state does not exist at the splitter blades, this is not due to the splitter blades. Similarly, this effect was not reflected in the results of the splitter blades of other types of pumps[6,7,23]. And the risk of blood damage from low velocity is much lower than high velocity. Overall, the PIV experiment reflects the good flow inside the pump. In the impeller region, the occurrence of abnormally high velocity was avoided, and no obvious flow characteristics that caused excessive blood damage were found.

There were several limitations to this study. First, the study did not evaluate optimization effects through blood experiments. The reason is that at present, our blood pump manufacturing and blood experimental technology are not enough, and the effects of materials, surfaces, and experimental operations on the results cannot be ruled out well. Besides, many scholars' studies have reflected that it is difficult to achieve a quantitative analysis of this degree of hemolytic difference in blood experiments [22,23]. In future research, we will improve technology and methods and strive to determine the optimization effect through blood experiments. Secondly, this study of splitter blades is only aimed at one type of axial flow 
blood pump. This is not comprehensive enough, but it is also a meaningful exploration and structural improvement. In future research, we will extend to other types, such as centrifugal pumps, to achieve more comprehensive applications.

\section{Conclusion}

The design of the blood pump is demanding, and it is necessary to consider both the hydraulic performance and hemolytic damage indicators. In this study, structural parameter characteristics analysis and parameter optimization were performed for splitter blades of blood pumps. Combining CFD simulation and neural network methods and conducting hydraulic cycle experiments and micro PIV experiments. The following conclusions and results were obtained:

1. A multi-parameter and multi-objective performance prediction model combining CFD and neural networks is established, which can assist in the prediction between parameters and performance in the design;

2. Analyze the performance of several core parameters of splitter blades, and the results reflect the potential of splitter blades to improve the performance of blood pumps $\rrbracket$

3. Through CFD, neural network, and PIV experiments and hydraulic experiments, a set of flow blade design parameters are obtained, which can improve the hydraulic performance of the blood pump and avoid increasing the risk of blood damage.

The research results provided an effective method and ideas for the structural improvement of the blood pump. The proposed method combining CFD and neural networks is helpful in improving the efficiency and reliability of multi-parameter and multi-target research on blood pumps.

\section{Methods}

\section{Blood pump and splitter blade}

The optimized design of the splitter blades is aimed at an axial blood pump with splitter blades. The main structure of the blood pump was previously designed through theory, CFD, and experiments. Figure 7 is the three-dimensional structure of the blood pump. The main structure includes a front diffuser, impeller, and back diffuser. A permanent magnet is installed inside the impeller, and an external magnetic driving device generates an alternating magnetic field to drive the impeller. The high rotation speed of the impeller can generate the fluid energy required for blood circulation, but the complicated flow that follows will bring the risk of hemolysis, so the structural design of the impeller is the most critical issue affecting the application.

Splitter blades are auxiliary blades on the impeller, which are located between every two main blades. The shape and thickness of the splitter blades are consistent with the main blade, but its length is shorter than the main blade. The splitter blades are equivalent to increasing the total length of the blades and 
subdividing the larger flow channels between the main blades. Therefore, the splitter blades can improve the hydraulic performance of the pump and make the flow between the main blades more stable. However, the blood pump is a device applied in the blood environment. The design of the blood pump needs to consider both hydraulic performance and blood damage, and the structure design has more stringent requirements. In this study, the structural optimization design of the splitter blade takes into account three core parameters, which are the number of blades, axial length, and circumferential offset.

Parameter 1 is the number of split blades. A larger number of blades can have a stronger effect on the fluid, but an excessive amount will make the flow channel too narrow, which may cause blockage of the fluid and blood damage. Parameter 2 is the axial length ratio of splitter blades, which is the ratio of the axial length of the splitter blade to the axial length of the main blade. Longer splitter blades can have a sufficient effect on the fluid, but it is necessary to control the arrangement of the splitter blades in a position with enough flow channel space. Parameter 3 is the circumferential offset of the splitter blade, which is the offset angle of the splitter blade to the back of the main blade. Splitter blades are generally offset towards the back of the main blade. This is to improve the unevenness of the circumferential velocity in the flow channel.

\section{Neural network method}

The optimal design of blood pump splitter blades has the characteristics of multi-parameters, multiobjectives, and non-linearity. The traditional design method has the disadvantages of a long cycle and inaccuracy. BP neural network is a multilayer network trained according to the error backpropagation algorithm. It is a machine learning method that simulates biological nerves. The sample data is used to train the

model to modify the network weights and thresholds, so that the error function decreases in the direction of the negative gradient, thereby improving the reliability of the output results. In application, this method is suitable for dealing with multiple fuzzy factors and non-linear relationships, which meets the optimization design requirements of the splitter blades. In this study, the mapping relationship between parameters and performance was established by this method to achieve performance prediction under corresponding parameters. Figure 8 shows the structure of the BP network established in this study.

The input layer has 3 neural units, which correspond to the number of blades, axial length ratio, and circumferential offset, respectively. There are two neural units in the output layer, which correspond to the pressure head and the hemolytic prediction index, respectively. The research determined the analysis range of the input parameters through theoretical analysis and simulation calculations. The relationship between the input and output of the neural network model is as follows:

$$
\left.\begin{array}{l}
x_{1} \\
x_{2} \\
x_{3}
\end{array}\right\} \Rightarrow\left\{\begin{array}{l}
f_{1}\left(x_{1}, x_{2}, x_{3}\right) \\
f_{2}\left(x_{1}, x_{2}, x_{3}\right)
\end{array}\right.
$$


In this equation, $f_{1}$ and $f_{2}$ are the output, which is the pressure head and the hemolytic prediction index, respectively. $x_{1}$ is the number of blades, and its analysis range is 0 to $4 . x_{2}$ is the axial length ratio, which refers to the ratio of the axial length of the splitter blades to the axial length of the main blade. The analysis range of $x_{2}$ is 0.3 to $0.7 . x_{3}$ is the degree of circumferential offset. It refers to the angle at which the splitter blades are offset to the back of the main blade. Its analysis range is $-15^{\circ}$ to $30^{\circ} . x_{3}$ is the circumferential offset, which is the degree to which the splitter blades are offset toward the back of the main blade. The analysis range of $x_{3}$ is $-15^{\circ}$ to $30^{\circ}$.

CFD analysis

CFD simulation was used to predict the performance of the 3D model with different parameters. The simulation uses ANSYS CFX 17.0 software (ANSYS, Inc., Canonsburg, PA, USA). Because the internal flow channel of the blood pump is distorted, the grid uses wall prism layer grids and unstructured grids. An independence analysis was performed on the grids, and the number of grids was determined to be about 2.5 million. The model is divided into three areas: front diffuser, impeller, and back diffuser. The impeller area is the rotation area, and the Frozen Rotor interface is used to achieve the connection between the regions.

In the simulation, blood is considered as an incompressible single-phase Newtonian fluid, which is a common and reliable simplification in blood pump calculations. The simulation uses the SST k-w model, which has good adaptability to complex turbulent flows. The boundary conditions of the inlet and outlet are flow inlet and pressure outlet, respectively. The Simplec algorithm was used to solve the pressurevelocity coupling equation, and the convergence accuracy reached $10^{-5}$. In the results, the pressure head was calculated from the pressure difference between the inlet and outlet, and the hydraulic performance of the model was evaluated with this head.

The shear stress in the blood pump flow field will cause hemolytic damage. In the design of the splitter blades, it is necessary to ensure that no additional hemolysis will occur. The blood test of the prototype has the problems of a long cycle and high cost. And there are many factors that are difficult to control, which will affect the accuracy of the blood experiment, so the CFD simulation is generally used to calculate the hemolysis index in the design stage [18].

$$
\tau=\left[\frac{1}{6} \Sigma\left(\tau_{i i}-\tau_{j j}\right)^{2}+\sum \tau_{i j}^{2}\right]^{0.5}
$$

The calculation of the hemolysis index is based on the classic hemolysis index model proposed by Giersiepen [19]. Five hundred random streamlines were extracted from the simulation results, and the hemolytic index was calculated by the shear stress loading of the streamlines, and their average value was used as the model result.

$$
D=d H b / H b(\%)=3.62 \times 10^{-7} t^{0.785} \tau^{2.416}
$$


In this equation, $D$ is the blood damage index, which is an estimate of the proportion of free hemoglobin. $\tau$ is the shear stress (Pa), and $t$ is the exposure time (s) of the shear stress. $H b$ is the total hemoglobin concentration, $d H b$ is the released hemoglobin concentration.

\section{Experiment}

This study used hydraulic and micro PIV experiments. The purpose of the hydraulic experiment is to obtain the hydraulic performance of the blood pump prototype. The performance indicators include flow rate and pressure head. Moreover, the accuracy of the CFD simulation results is verified by the results of hydraulic experiments. The hydraulic experimental device includes pump prototype, external magnetic drive system, pressure gauge, ultrasonic flow meter, and thermostatic water bath. The experiment uses a $38 \%$ volume glycerol solution, which is close to the fluid properties of blood under normal temperature conditions.

The rotor (impeller and diffuser) of the blood pump prototype is made of titanium alloy. The impeller is equipped with N48 Ru-Fe-B permanent magnets. The alternating magnetic force generated by the magnetic drive system makes the impeller rotate, and the maximum speed can reach about $12000 \mathrm{rpm}$. There are two types of impellers for hydraulic experiments: prototype impellers without splitter blades and impellers with optimized parameter splitter blades.

In addition, micro PIV experiments were used to measure and analyze the flow inside the pump. PIV is a non-contact experimental technique for flow velocity measurement. This method calculates the instantaneous velocity by obtaining the displacement of the tracer particles in a short time. When the followability of the tracking particles is good enough, the particle velocity can be regarded as the flow field velocity[20,21]. The PIV system used in this experiment was produced by TSI. The camera is Zyla 5.5, which can capture up to 100 instantaneous particle images in 1 second.

To meet the needs of PIV experiments, the pump casing is made of highly transparent plexiglass material. Besides, a square water tank is arranged on the outside of the pump to reduce the influence of the refractive index. The water tank uses the same high-permeability plexiglass material as the pump casing. In the experiment, both the water tank and the pump are filled with the same glycerin solution. Since the organic glass material and the glycerin solution have a close refractive index, the influence of the refractive index on the result can be reduced. The tracer particles were rhodamine fluorescent particles with a diameter and density of $7.0 \mu \mathrm{m}$ and $1.1 \mathrm{~kg} / \mathrm{m}^{3}$, respectively. The reflection on the metal surface is filtered by a $560 \mathrm{~nm}$ camera filter. Figure 9 (a) is the PIV experiment system. Figure 9 (b) is the particle image and the method of particle velocity calculation. In the calculation, identify the same particle in two photos with $\Delta t$ interval, and obtain the velocity by the particle's movement position. The $\Delta t$ of this PIV experiment is set to 100 microseconds. Because $\Delta t$ is sufficiently small, the result can be regarded as a transient velocity.

$$
v_{x}=\frac{d x(t)}{d t}=\frac{x(t+\Delta t)}{\Delta t}
$$




$$
v_{y}=\frac{d y(t)}{d t}=\frac{y(t+\Delta t)}{\Delta t}
$$

\section{Abbreviations}

CFD: computational fluid mechanics; PIV: particle image velocimetry; VAD: Ventricular assist device.

\section{Declarations}

Acknowledgements

The authors are grateful to all study participants.

Authors' contributions

Conceptualization, Yu and Tan; software, Yu and Wang; validation, Yu and Wang; formal analysis, Yu; investigation, Yu and Wang; writing-original draft preparation, Yu writing-review and editing, Yu and Tan; supervision, Tan. All authors have read and agreed to the published version of the manuscript.

Funding

This research was supported by National Natural Science Foundation Project of China, grant number 51475477 and 31670999, and Postgraduate Independent Research and Innovative Project of Central South University, grant number $2018 z z t s 021$.

Availability of data and materials

The data sets used and/or analyzed during the current study are available from the corresponding author on reasonable request.

Ethics approval and consent to participate

Not applicable.

Consent for publication

Not applicable.

Competing interests

The authors of this article declare that there is no conflict of interest.

\section{References}


1. Kirklin J K, Naftel D C, Pagani F D, et al. Seventh INTERMACS annual report: 15,000 patients and counting. Journal of Heart \& Lung Transplantation. 34, 1495-1504 (2015)

2. Bockeria, L. A. , Bockeria, O. L. , Selishchev, S. V. , Telyshev, D. V. , \& Glushko, L. A. . Experimental determination of the normalized index of hemolysis for the sputnik implantable pediatric rotary blood pump. Biomedical Engineering. 50(3), 416-419 (2017)

3. Nosé, Y, Yoshikawa, M. , Murabayashi, S. , \& Takano, T. . Development of rotary blood pump technology: past, present, and future. Artificial Organs. 24(6), 412-420 (2015)

4. Bludszuweit $\mathrm{C}$. Three-dimensional numerical prediction of stress loading of blood particles in a centrifugal pump. Artif Organs. 19, 590-603 (1995)

5. Bludszuweit C. Model for a general mechanical blood damage prediction, Artif Organs. 19, 583-589 (1995)

6. Shouqi, Y. , Jinfeng, Z. , Jianping, Y., Youshi, H. E. , \& Yuedeng, F. U. . Effects of splitter blades on the law of inner flow within centrifugal pump impeller. Chinese Journal of Mechanical Engineering. 20(5), 59-63 (2007)

7. Shigemitsu T,Fukutomi J,Kaji K, et al. Unsteady Internal Flow Conditions of Mini-Centrifugal pump with Splitter Blades. Journal of Thermal Science. 22, 86-91 (2013)

8. Apel J, Paul R, Klaus S, et al. Assessment of Hemolysis Related Quantities in a Micro axial Blood Pump by Computational Fluid Dynamics. Artificial Organs. 25, 341-347 (2015)

9. Noor $\mathrm{M} \mathrm{R}, \mathrm{Ho} \mathrm{CH}$, Parker K H, et al. Investigation of the Characteristics of HeartWare HVAD and Thoratec HeartMate II Under Steady and Pulsatile Flow Conditions. Artif Organs. 40, 549-560 (2016)

10. Taskin, M. E. , Fraser, K. H. , Zhang, T. , Gellman, B. , \& Wu, Z. J. Computational characterization of flow and hemolytic performance of the ultra)mag blood pump for circulatory support. Artificial Organs. 34(12), 1099-1113 (2010)

11. Graefe R, Henseler A, Steinseifer U, Multivariate Assessment of the Effect of Pump Design and Pump Gap Design Parameters on Blood Trauma. Artificial Organs. 40, 568-576 (2016)

12. Takano, T. , Schulte-Eistrup, Sebastian, Yoshikawa, M. , Nakata, Kin-ichi, Kawahito, S. , \& Maeda, T. , et al. Impeller design for a miniaturized centrifugal blood pump. Artificial Organs. 24(10), 821-825 (2015)

13. Pei, J., Wang, W., Osman, M. K., \& Gan, X. Multiparameter optimization for the nonlinear performance improvement of centrifugal pumps using a multilayer neural network. Journal of Mechanical Science and Technology. 33(6), 2681-2691 (2019)

14. Zhao, W. , Sheng, J. , Yang, J. , \& Song, Q. Optimization design and experiment of centrifugal pump based on cfd. Transactions of the Chinese Society of Agricultural Engineering. 31(21), 125-131 (2015)

15. Rong, L. U. , Jianping, Y. , Yanjun, L. I. , \& Hongying, J. Automatic optimization of axial flow pump based on radial basis functions neural network and cfd. Journal of Drainage and Irrigation Machinery Engineering. 35(6), 481-487 (2017) 
16. Minggao, T. Energy characteristics prediction of centrifugal pumps based on artificial neural network. Transactions of the Chinese Society for Agricultural Machinery. 33, 12-22 (2010)

17. An, Z. , Zhounian, L. , Peng, W. , Linlin, C. , \& Dazhuan, W. Multi-objective optimization of a low specific speed centrifugal pump using an evolutionary algorithm. Engineering Optimization. 48(7), 1251-1274 (2016)

18. Herbertson L H, Olia S E, Daly A, et al. Multilaboratory Study of Flow-Induced Hemolysis Using the FDA Benchmark Nozzle Model. Artificial Organs. 39, 237-248 (2015)

19. Giersiepen $M$, Wurzinger $L$, Opitz R, Reul H. Estimation of shear stress-related blood damage in heart valve prostheses - in vitro comparison of 25 aortic valves. Int J Artif Organs. 13, 300-306 (1990)

20. Su, B. , Chua, L. P. , \& Wang, X. Validation of an axial flow blood pump: computational fluid dynamics results using particle image velocimetry. Artificial Organs. 36(4), 359-367 (2011)

21. Zou, Y. , Ye, S. S. , Wang, Y. D. , \& Fei, W. Y. Cfd simulation and piv measurement of liquid-liquid twophase flow in pump-mix mixer. Journal of the Taiwan Institute of Chemical Engineers. 60, 15-25 (2015)

22. Hariharan, P. , Gavin A. D’Souza, Horner, M. , Morrison, T. M. , \& Myers, M. R. Use of the fda nozzle model to illustrate validation techniques in computational fluid dynamics (cfd) simulations. PLOS ONE. 12(6), e0178749 (2017)

23. Yang, X. , Gui, X. , Huang, H. , Shen, Y. , Yu, Z. , \& Zhang, Y. Particle image velocimetry experimental and computational investigation of a blood pump. Journal of Thermal Science. 03, 68-74 (2013)

\section{Figures}




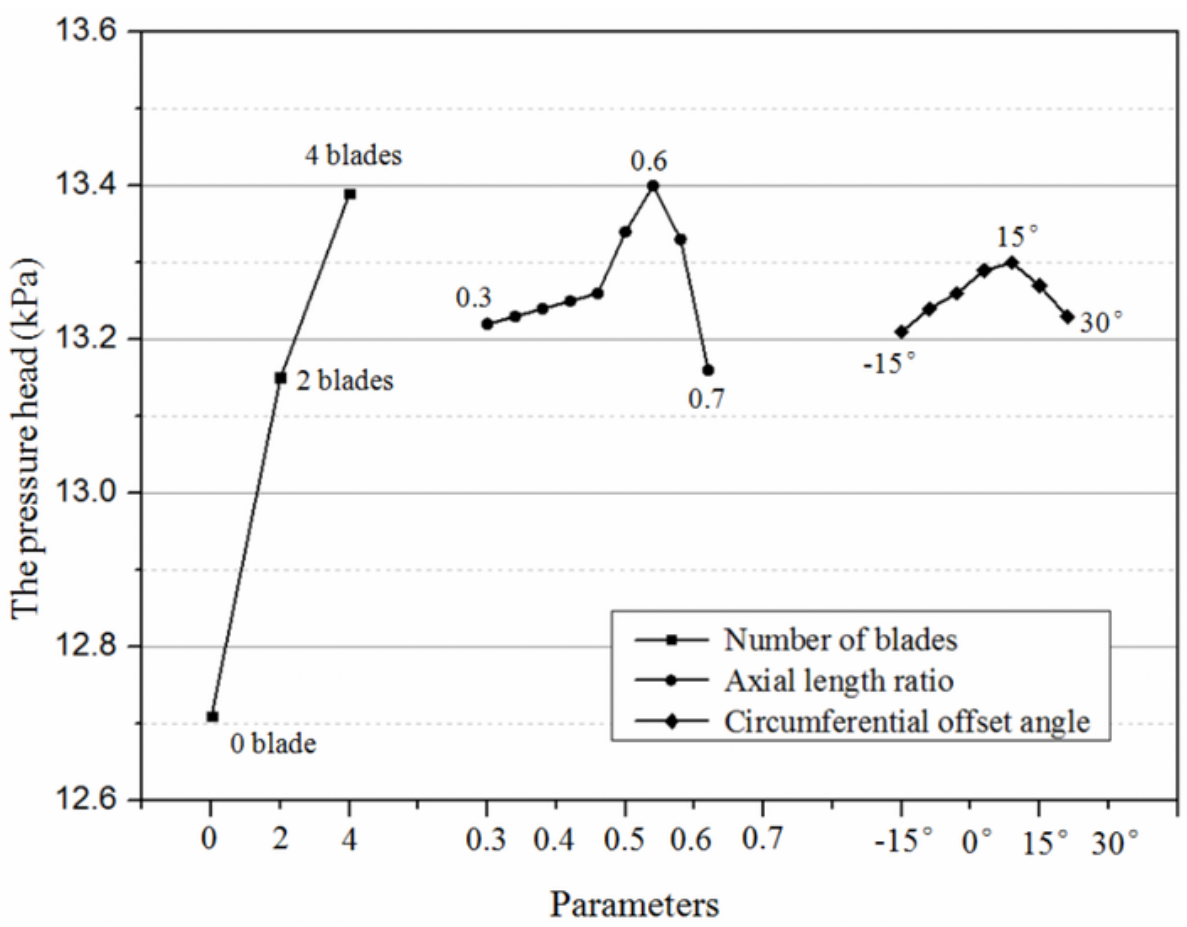

(a)

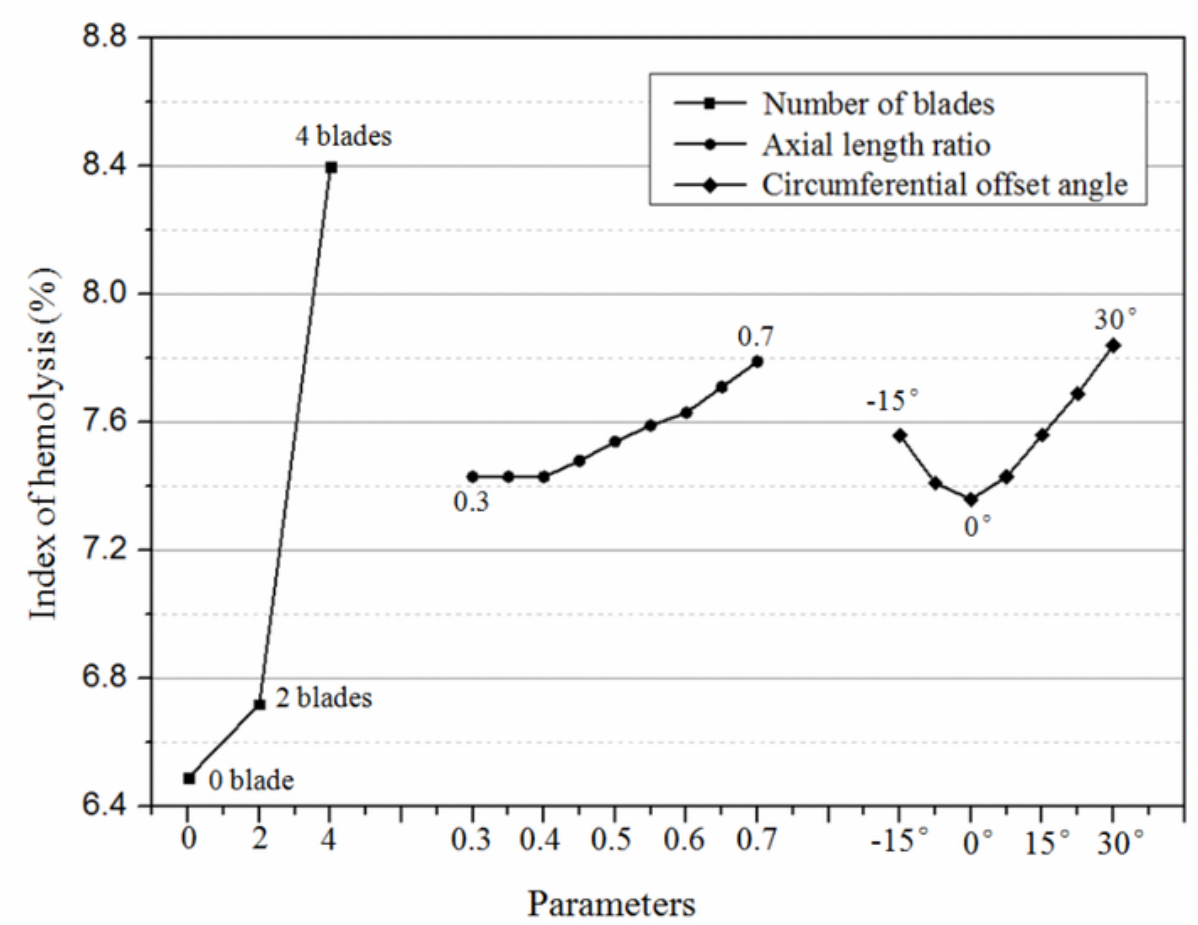

(b)

\section{Figure 2}

Average results of hydraulic head and hemolytic index under various parameters 
Neural network method

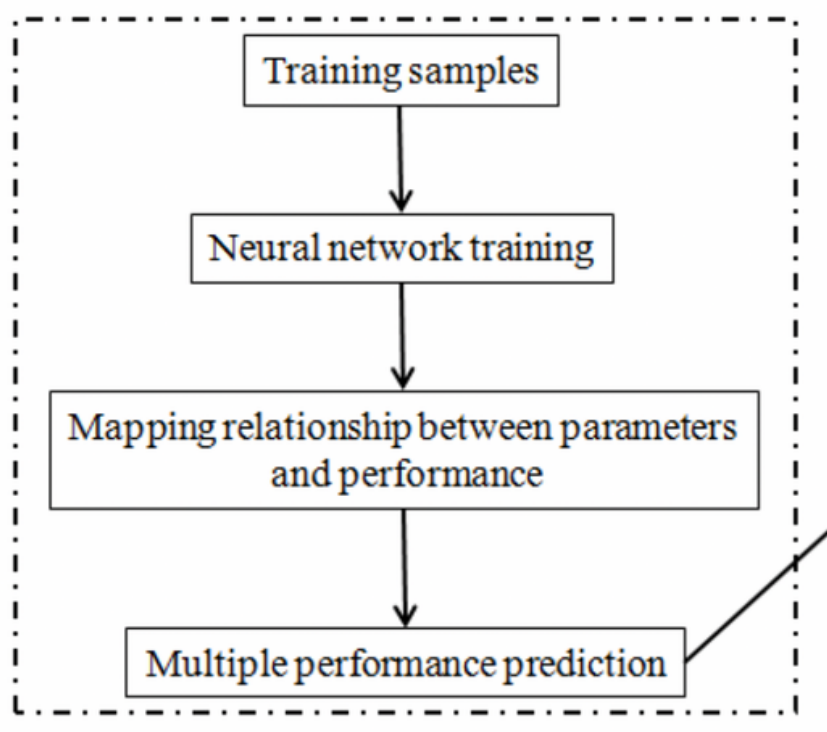

Multi-objective optimization of splitter blade parameters

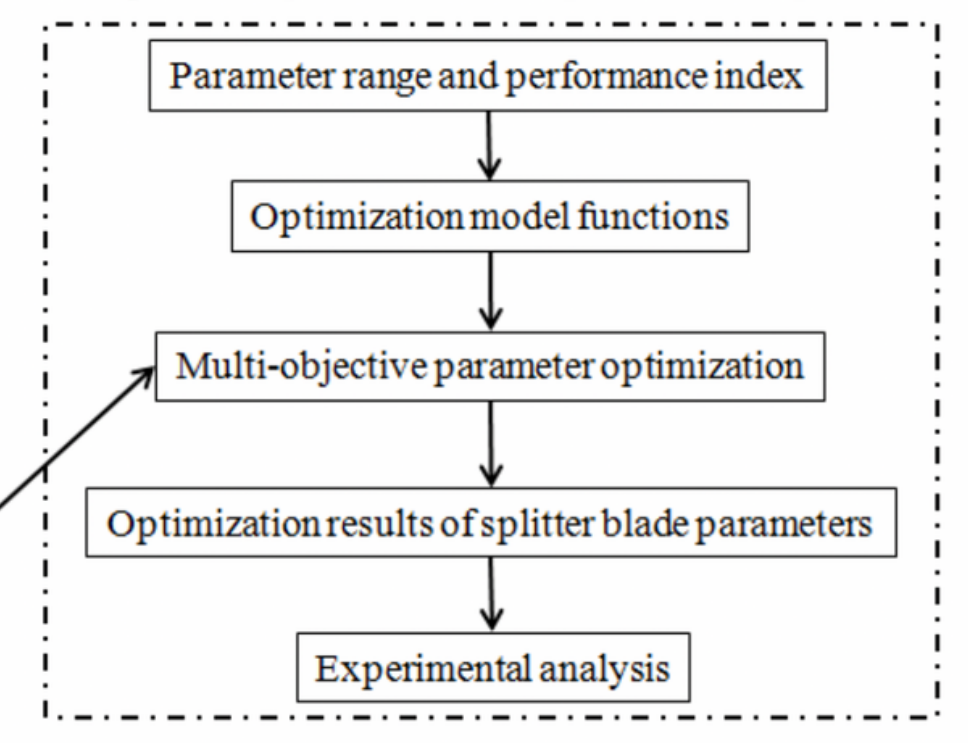

Figure 4

Parameter optimization process of splitter blades 


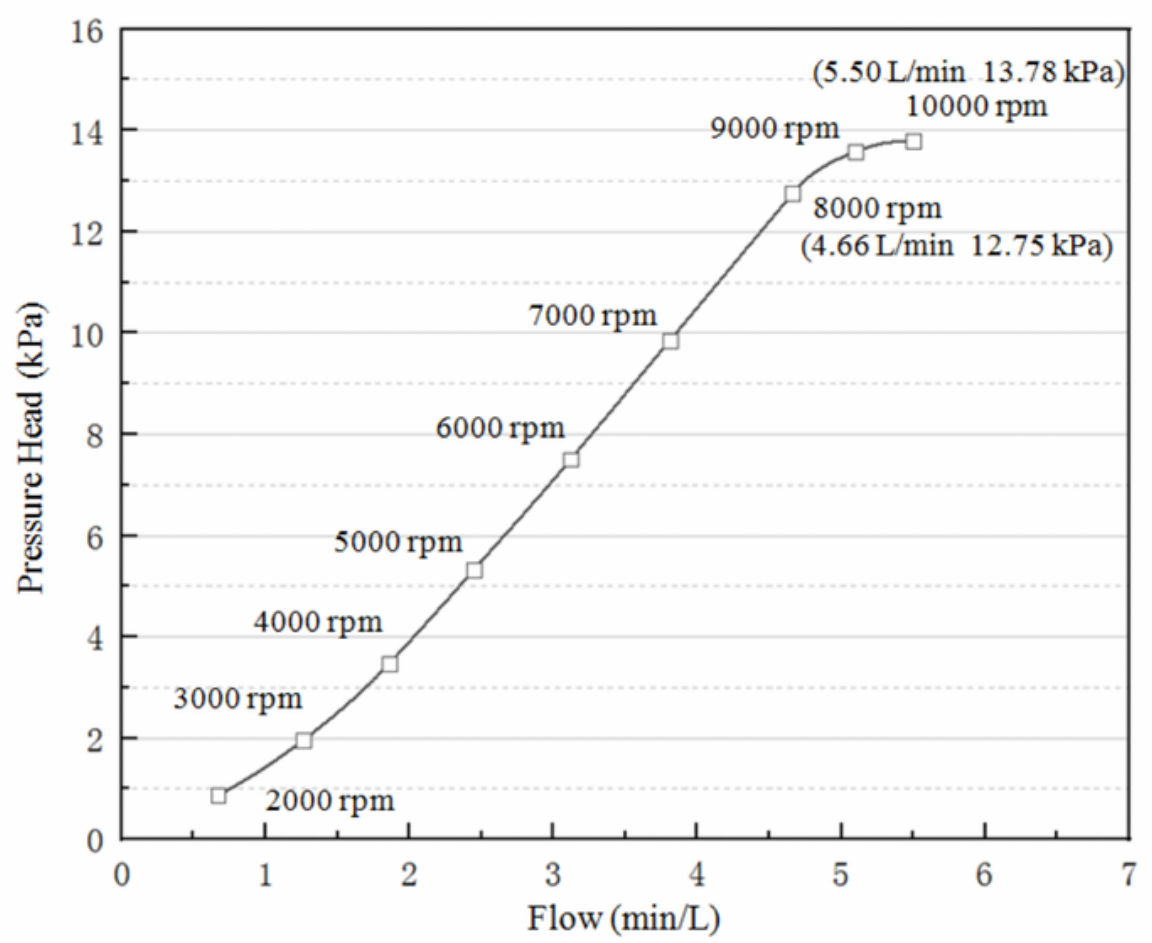

(a)

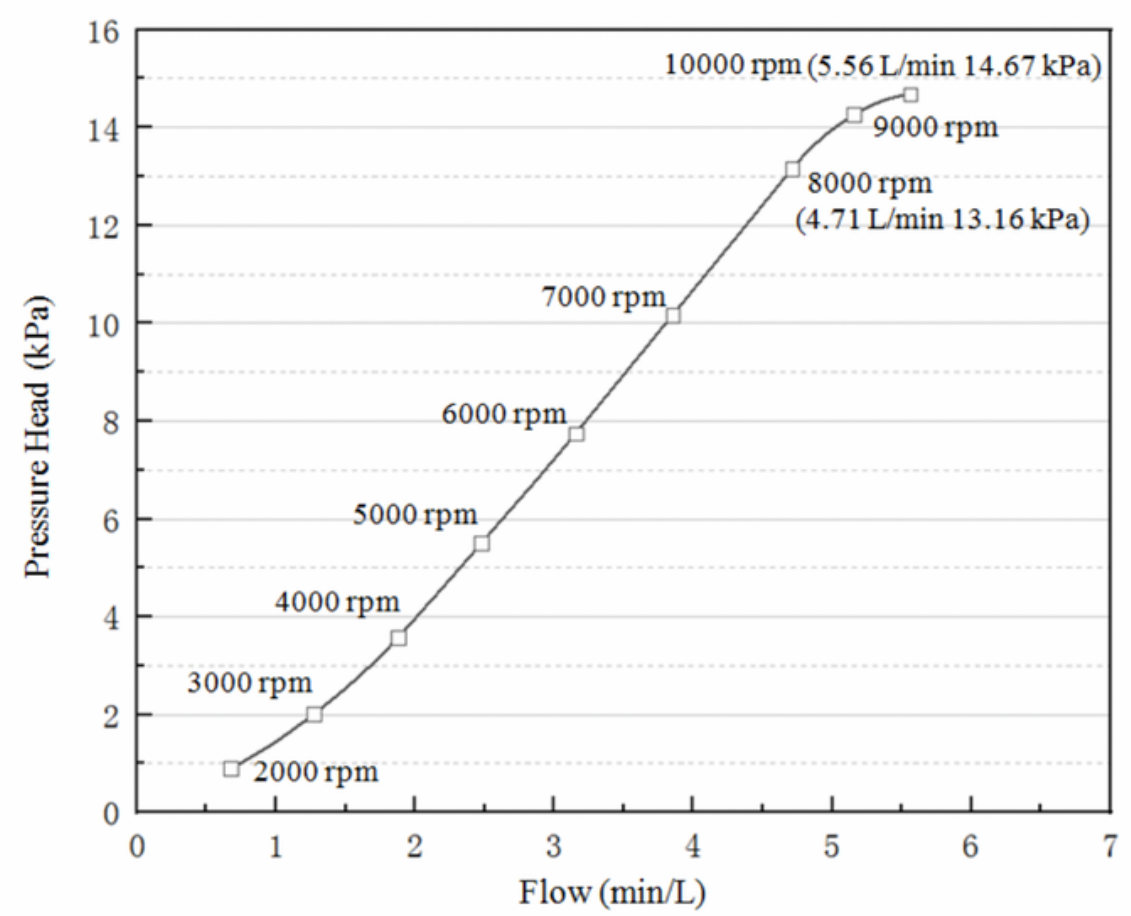

(b)

\section{Figure 5}

Experimental results of hydraulic performance (Figure (a) is the result of model-a without splitter blades, Figure (b) is the result of model-b with optimized splitter blades.) 


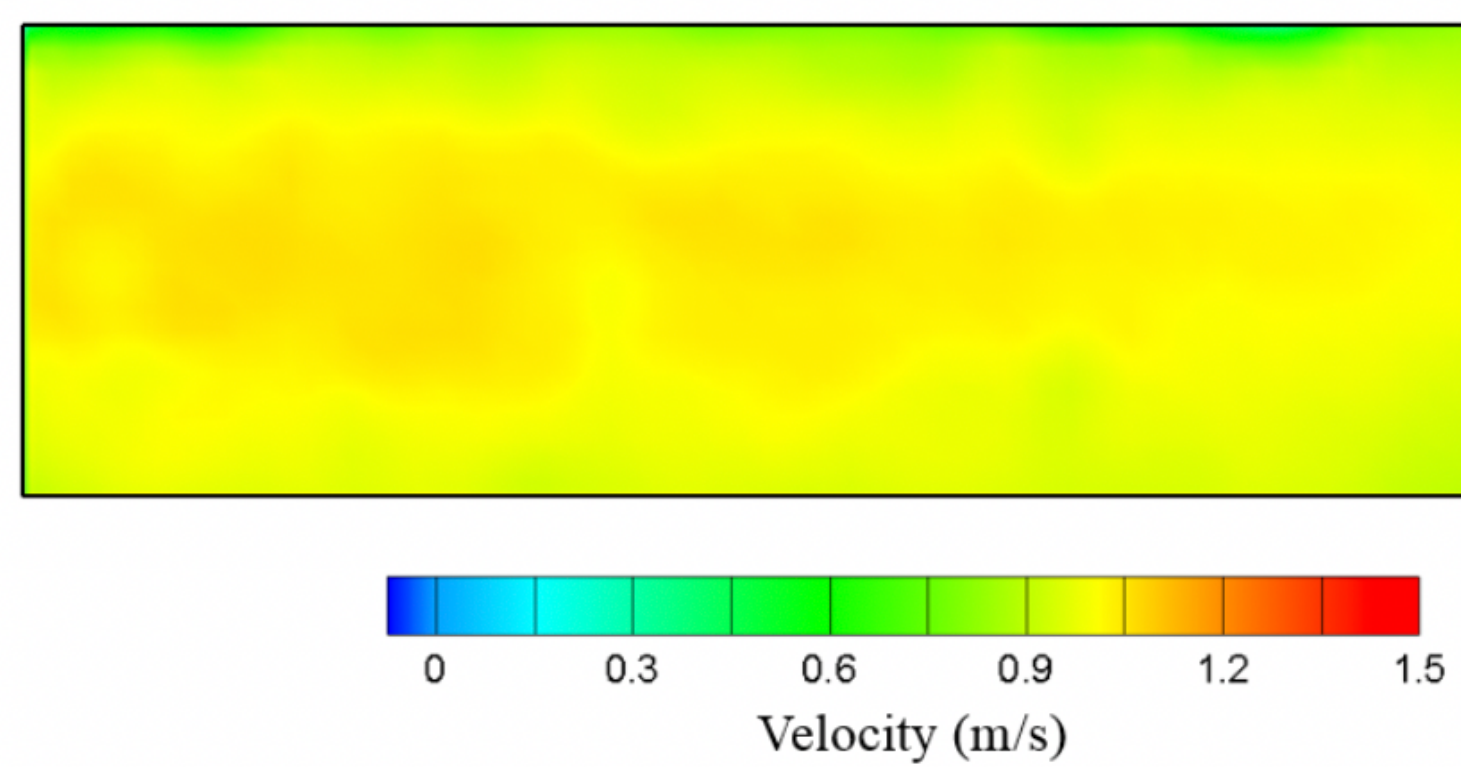

Figure 7

PIV results in front diffuser area
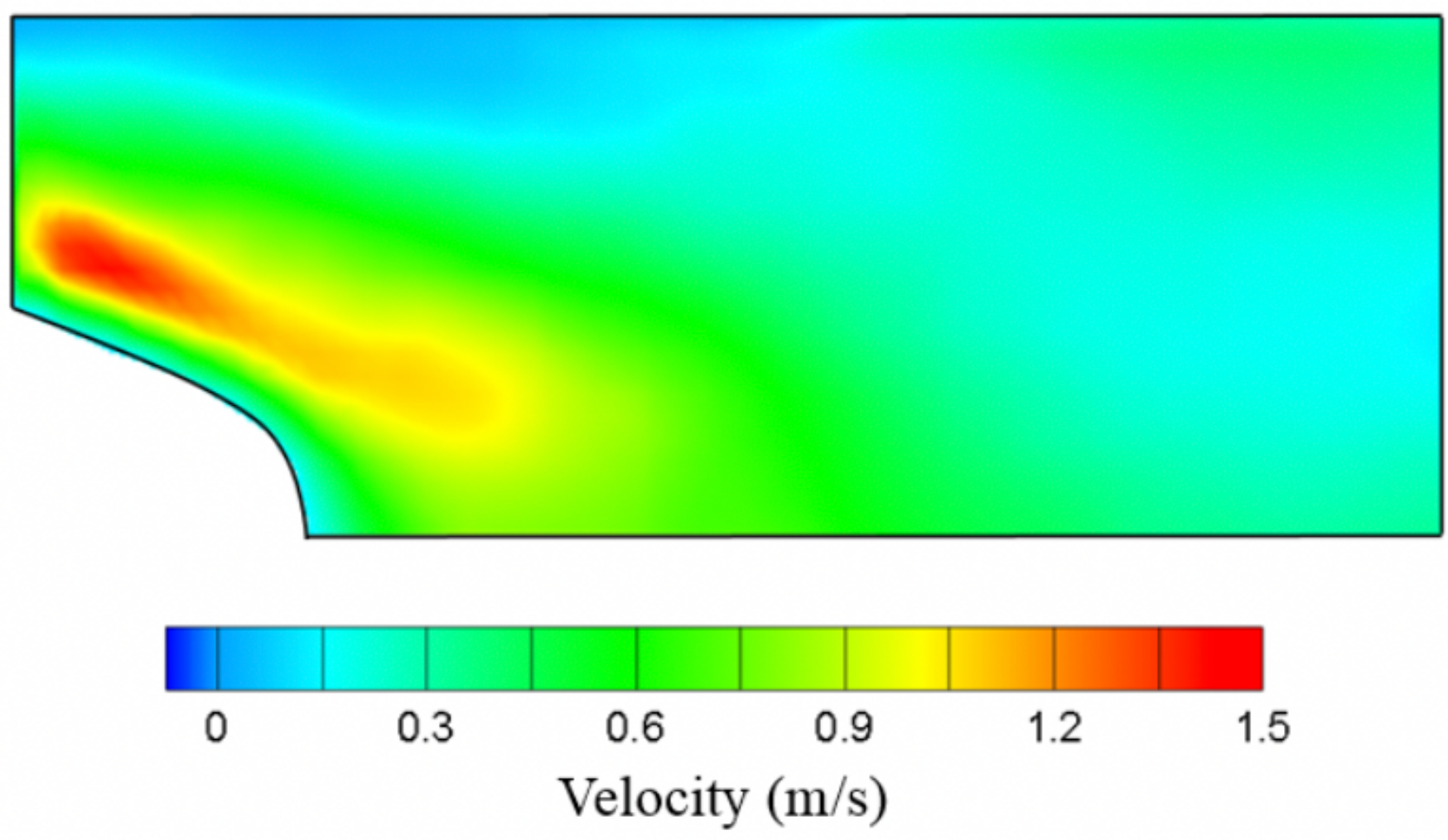

Figure 9

Results of PIV in the back diffuser area 


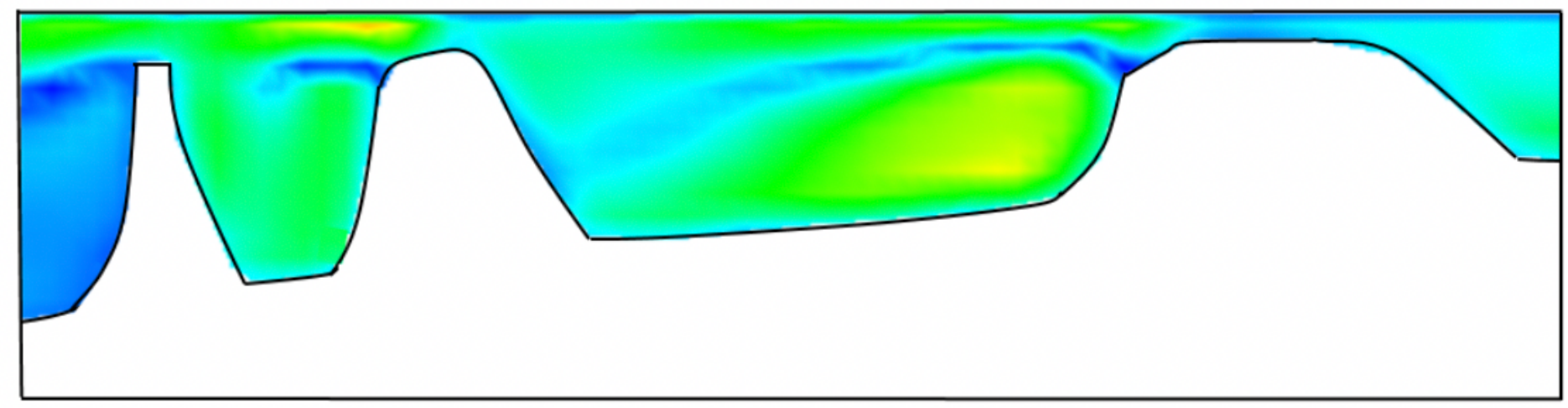

Figure (a)

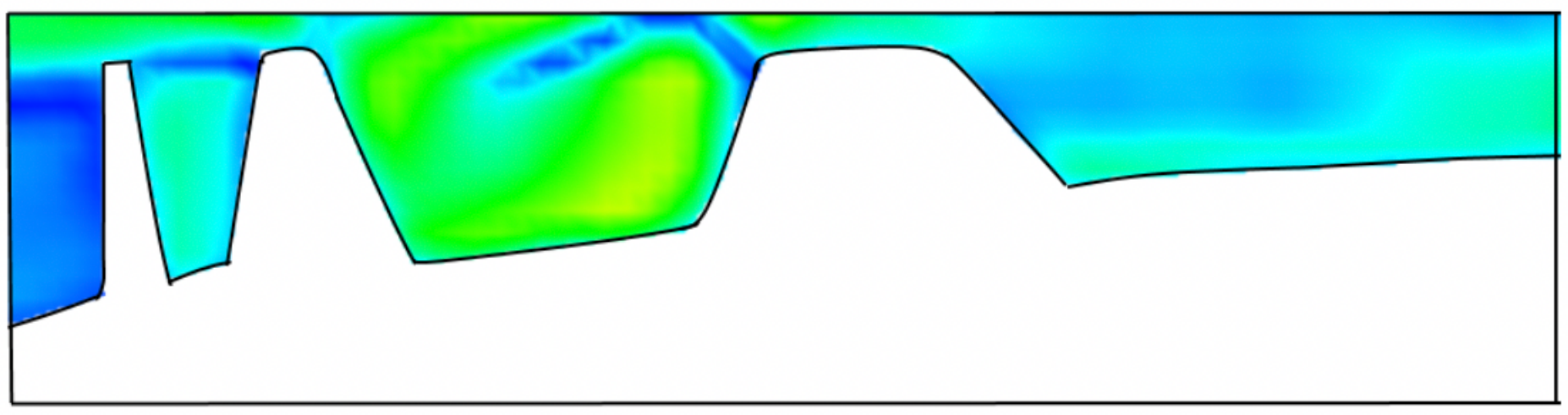

Figure (b)

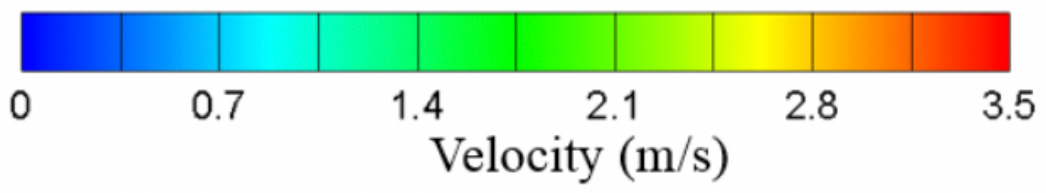

Figure 11

PIV results in the impeller region 


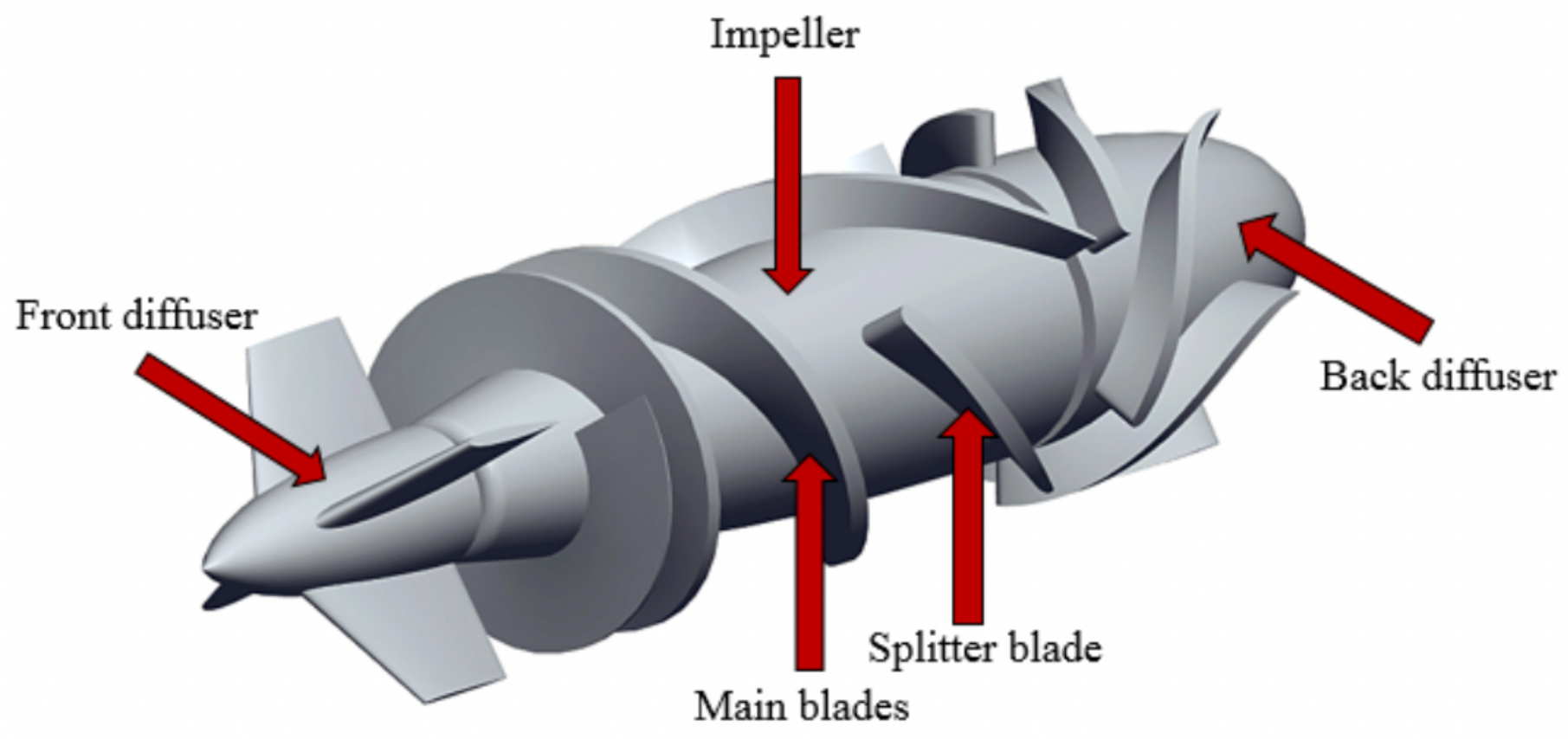

Figure 13

Rotor structure of axial flow blood pump

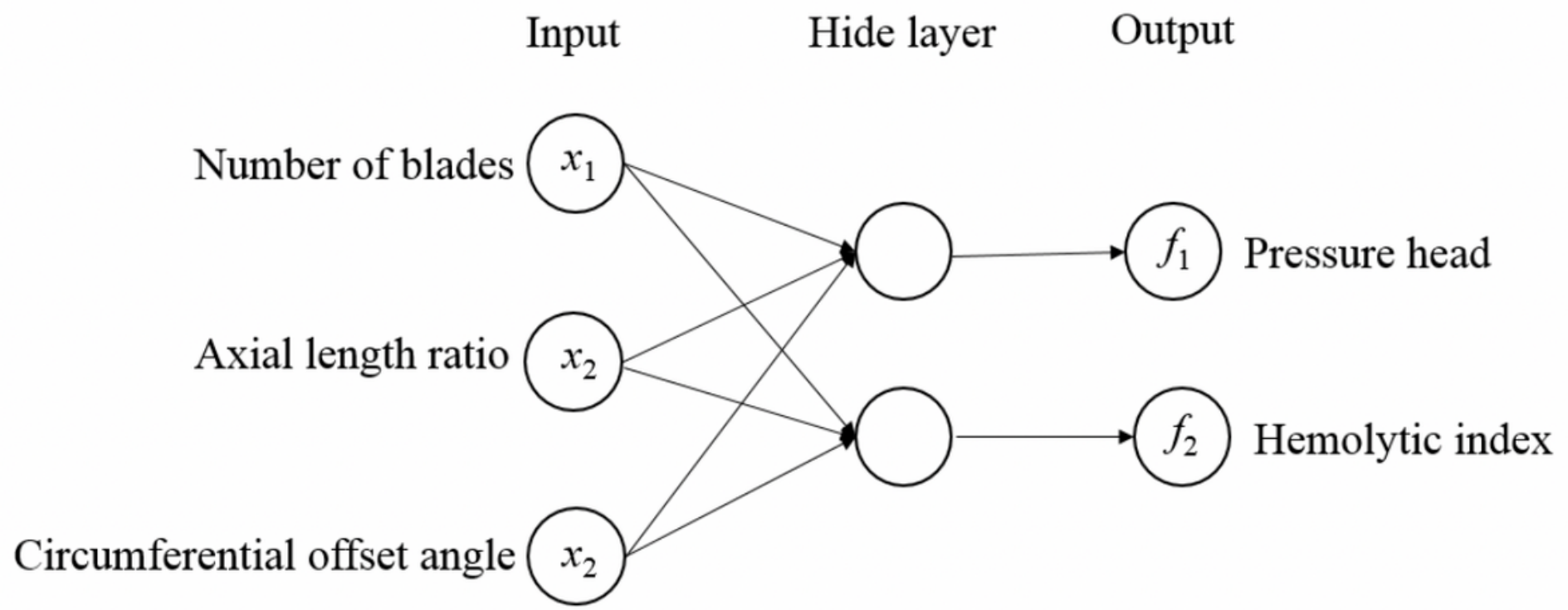

Figure 15

BP neural network model of blood pump shunt blade 


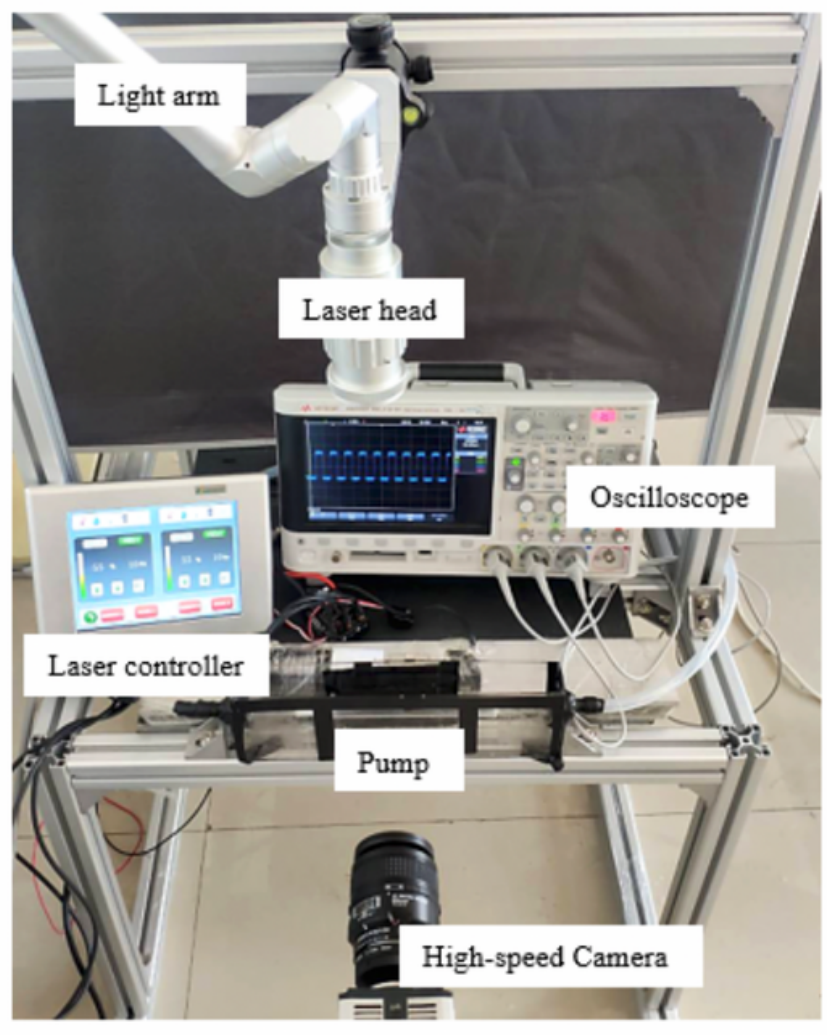

Figure (a)

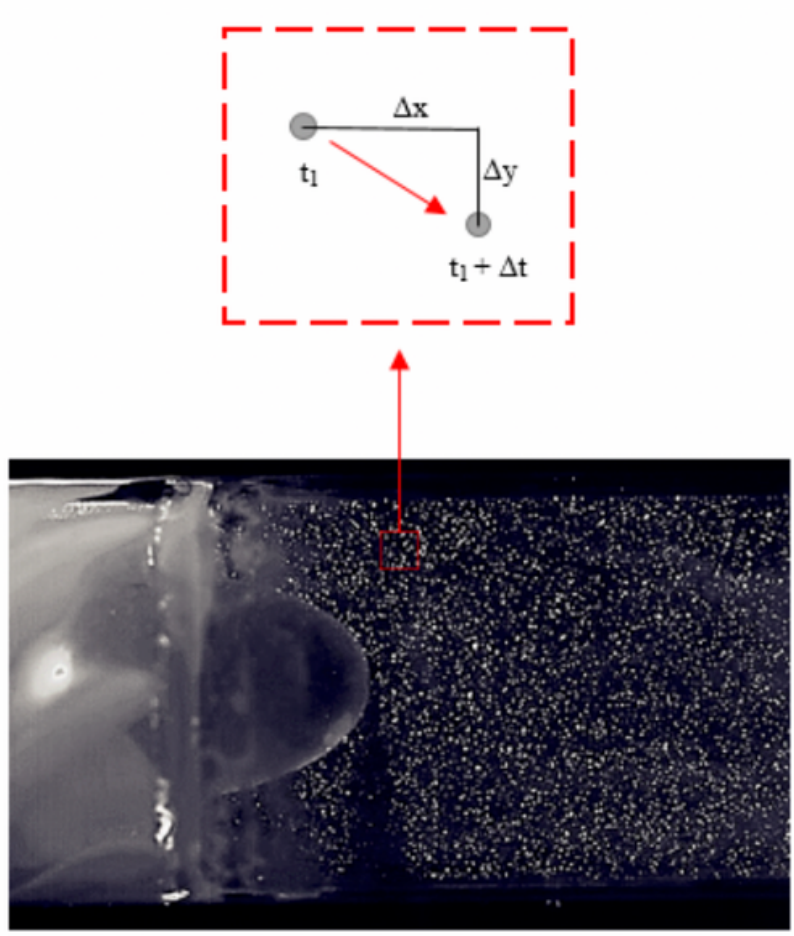

Figure (b)

\section{Figure 17}

PIV experimental system and particle images. (a) PIV experimental system; (b) Particle images of the back diffuser area. 